THE PARKES $2700 \mathrm{MHz}$ SURVEY: COUNTS OF THE SOURCES AND THEIR DISTRIBUTION ON THE SKY

\author{
J.V. Wa11 \\ Mullard Radio Astronomy Observatory, \\ Cavendish Laboratory, \\ Cambridge, England.
}

The Parkes 2700-MHz survey for extragalactic sources now extends over virtually the whole sky available to the 64-m telescope at galactic latitudes $|\mathrm{b}|>10^{\circ}$. Eleven parts of the catalogue have been published as Astrophysical Supplements of the Aust. J. Phys. (Numbers 19, 21, 26, $30,32,34$, and 39); part 11 and subsequent parts consist of re-surveys of some areas to fainter flux density limits. The current version of the composite catalogue for the $7.36 \mathrm{sr}$ covered (Fig. 1) contains about 7500 sources, together with the results of identification programmes and flux density measurements at other frequencies.

The survey was undertaken to examine the extent and nature of the population of "flat-spectrum" or "cm-excess" sources. The increase in representation of this type of source as the survey frequency is raised is clear from studies of sources detected both in this survey and in the NRAO 5000-MHz survey, and is well demonstrated in 'two spectral-index' diagrams (Wall 1975). The extent of the flat-spectrum population, which constitutes $\sim 40 \%$ of sources found at $2700 \mathrm{MHz}$ and $\sim 60 \%$ at $5000 \mathrm{MHz}$, makes it evident that the trials of surveyors at low frequencies - the survey, spectral observations, positions/identifications/redshifts, anisotropy/association searches, source-count

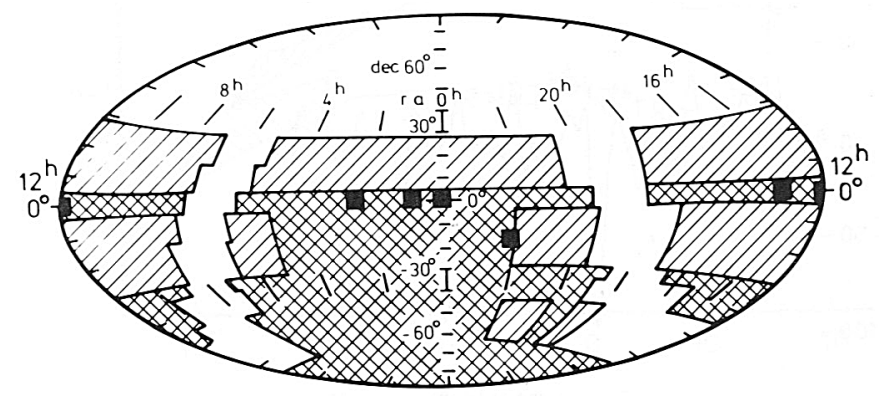

Fig. 1. The Parkes $2700 \mathrm{MHz}$ survey. Hatched areas are complete to 160 sources/sr, cross-hatched areas to $500-900$ sources/sr, and filled areas to 2500 sources/sr.

D. L. Jauncey (ed.). Radio Astronomy and Cosmology, 55-61. All Rights Reserved. Cop!right' 1977 by the IAU. 


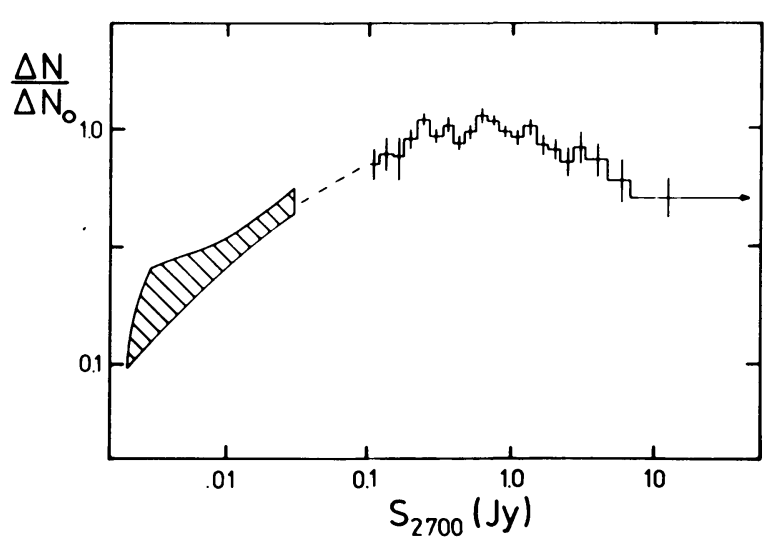

Fig. 2. The $2700 \mathrm{MHz} \mathrm{N}(\mathrm{S})$ relation.

compilation and analysis - must be endured by surveyors at high frequencies also. Now, however, the interpretation is harder because at least two source-populations are involved, so that there is even greater incentive to be certain of the observations and initial premises before proceeding. This contribution is therefore confined to the basic data, and considers the morphology of the source counts and the uniformity of source distribution over the sky.

\section{THE 2700-MHz N(S) RELATION}

Fig. 2 shows the 2700-MHz source count in relative differential form, extending down to $\mathrm{S}_{2700}=0.10 \mathrm{Jy}$ (2500 sources $\mathrm{sr}^{-1}$ ). (Details of the compilation will be presented elsewhere). The survey parameters are such that the confusion bias at 2500 sources $\mathrm{sr}^{-1}$ is far smaller than the statistical errors, so no such correction has been made. The faint end of the N(S) relation is delineated by a P(D) (background



Fig. 3. Two independent observations of the confusion limit along the same r.a. scan, shown displaced. 
deflection) analysis. The peculiarly-shaped area in Fig. 2 is from preliminary consideration of new data obtained at Parkes by D.J. Cooke and myself, and supersedes a previous determination (Wall and Cooke 1975) with which it is consistent. Some of the new data are shown in Fig. 3. These were obtained with the twin-beam system used for the survey, for which the receiver output is the difference between the signals received by the two beams. The independent observations of the same area show that the deflections are genuine and much larger than noise errors, and therefore the sampling of such scans yields a distribution of deflections due primarily to faint extragalactic sources; analysis of this sets limits on the $N(S)$ relation to a source density as high as one per beam area, or $2 \times 10^{5} \mathrm{sr}^{-1}$, corresponding to $\mathrm{S}_{2700} \sim 2 \mathrm{mJy}$.

Qualitatively the $N(S)$ relation of Fig. 2 is similar to the lowfrequency relations, showing an initial rise indicative of an integral slope steeper than the $-3 / 2$ (Euclidean) value, followed by a turnover and a fall at the lowest flux densities. The 2700-MHz count is smooth, in that fluctuations do not exceed those expected from the statistics of limited numbers. In particular there are no regions of abrupt changes in slope, which have been observed at both lower and higher frequencies (Jauncey 1975), and which have suggested to some authors that the counts do not have cosmological relevance. Qualitatively there is an obvious difference between the $2700-\mathrm{MHz}$ relation and those at lower frequencies in that the region of turnover is much broader at $2700 \mathrm{MHz}$. This effect, noted independently by Fomalont et al. (1974) and by Wal1 and Cooke (1975), is very apparent in Fig. 4, a collection

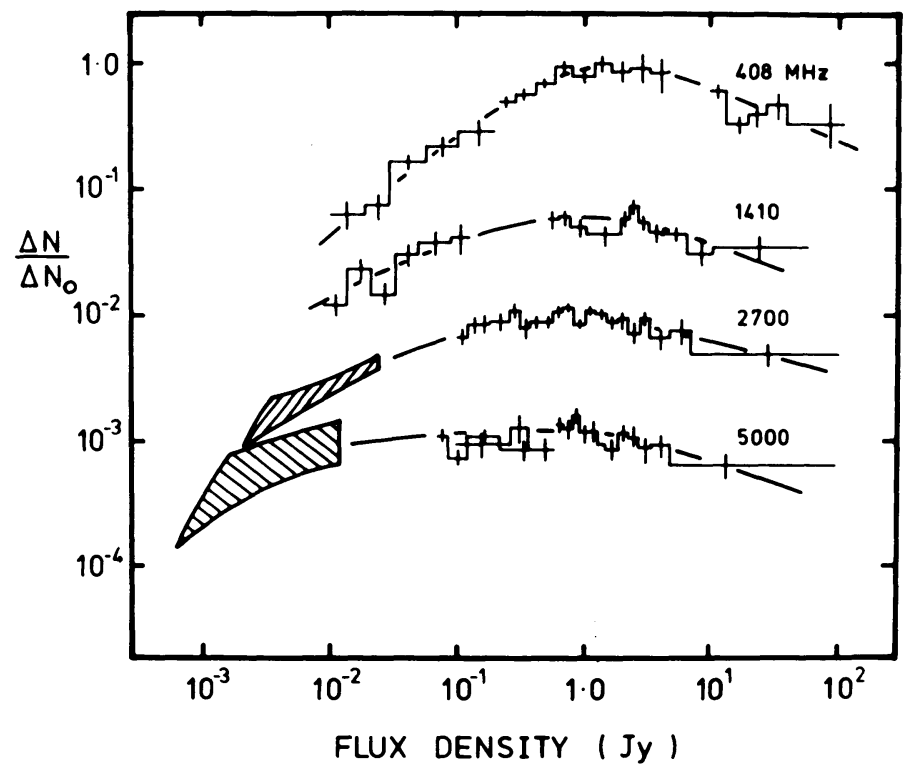

Fig. 4. N(S) relations at four frequencies; references to original data in Wall and Cooke (1975). 
of $N(S)$ relations at four frequencies. Included in this diagram is the preliminary result of a recent $\mathrm{P}(\mathrm{D})$ analysis at $5000 \mathrm{MHz}$ which provides striking confirmation of the trend, and shows that the relation at this frequency is close to Euclidean in slope over a range of three decades in flux density.

These results imply that the flat-spectrum sources favoured by high-frequency surveys have an $N(S)$ relation which differs from that for steep-spectrum sources. The effect is most marked at the faint end of the relations. Nevertheless it can be demonstrated directly at the bright end, using the 5000-MHz flux densities available for most sources of the $2700-\mathrm{MHz}$ survey with $\mathrm{S}_{2700}>0.35 \mathrm{Jy}$. Division of the sample at $\alpha_{2700} 000=0.5\left(S \propto v^{-\alpha}\right)$ yields the counts shown in Fig. 5. The flat-spectrum sources give a flatter count in accordance with Fig. 4, with the spectral-index - flux density dependence found by Wall (1972) and by Condon and Jauncey (1974), and with the earliest results obtained from the $2700 \mathrm{MHz}$ survey (Shimmins et al. 1968). The division at $\alpha_{2700}=0.5$ is of physical significance in that virtually all flat-spectrum sources thus defined have structures dominated by compact components, and in regions of low galactic obscuration, J.G. Bolton and B.A. Peterson have shown that most of these can be identified with QSOs.

Potentially the 2700-MHz count offers a powerful means of exploring the evolution of the flat-spectrum population, but its validity for cosmological exploration is clearly suspect if sources show serious anisotropy in their distribution over the sky.

\section{THE DISTRIBUTION OF THE SOURCES ON THE SKY}

In the course of the survey we obtained subjective impressions of areas disproportionately rich in flat-spectrum sources, and furthermore, we found the curious result of Fig. 6, wherein the first 2 sr of the survey produced both a significantly higher source density and a flatter count than the remaining $5 \mathrm{sr}$. It is impossible to attribute this result to instrumental effects, and it is important to note that the first $2 \mathrm{sr}$

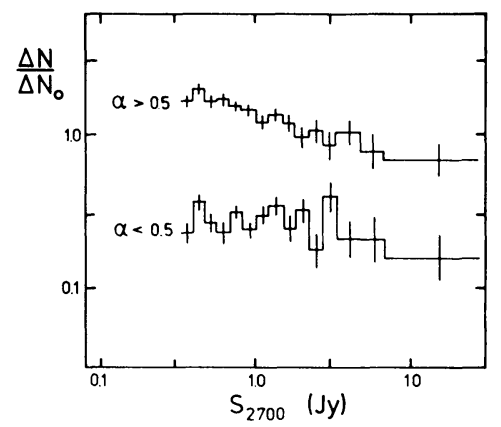

Fig. 5. Counts for steep-spectrum and flat-spectrum sources, displaced for clarity. 


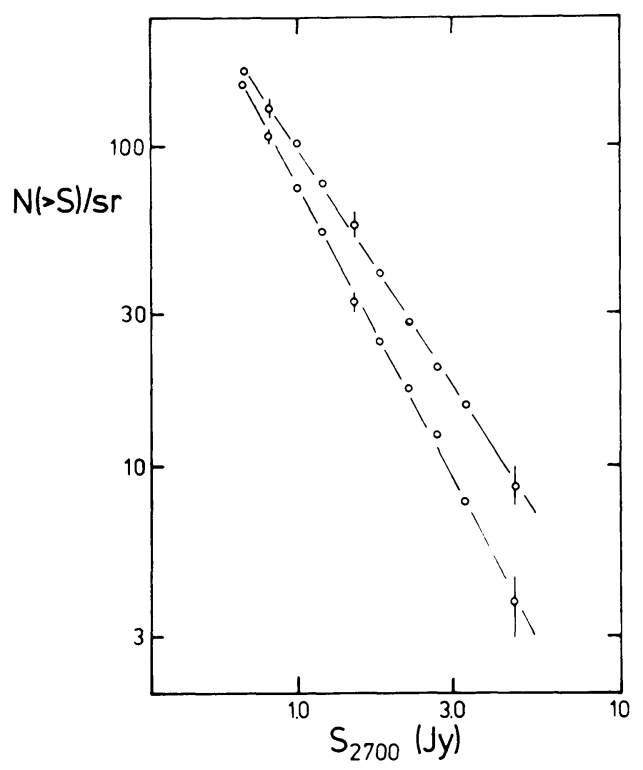

Fig. 6. Integral source counts for first 2 sr of survey (upper; maximum -likelihood slope $-1.48 \pm .08$ ) and remaining 5 sr (slope $-1.91 \pm .06$ ).

and the remaining $5 \mathrm{sr}$ are not contiguous areas, but are made up of regions typically $0.3 \mathrm{sr}$ in size and strewn all over the sky. Several analyses of source distribution were undertaken to investigate these effects.

On the largest possible scales, Yahil (1972) suggested that differences in the source counts between galactic hemispheres were present in several surveys, and results from the NRAO 5000-MHz survey (Pauliny-Toth and Kellermann 1972) added weight to the suggestion. The maximum-likelihood slopes of counts in the hemispheres from the full 7 sr of the $2700 \mathrm{MHz}$ survey $\left(\mathrm{S}_{2700}>0.7 \mathrm{Jy}\right)$ are as follows, with the numbers of sources in brackets:

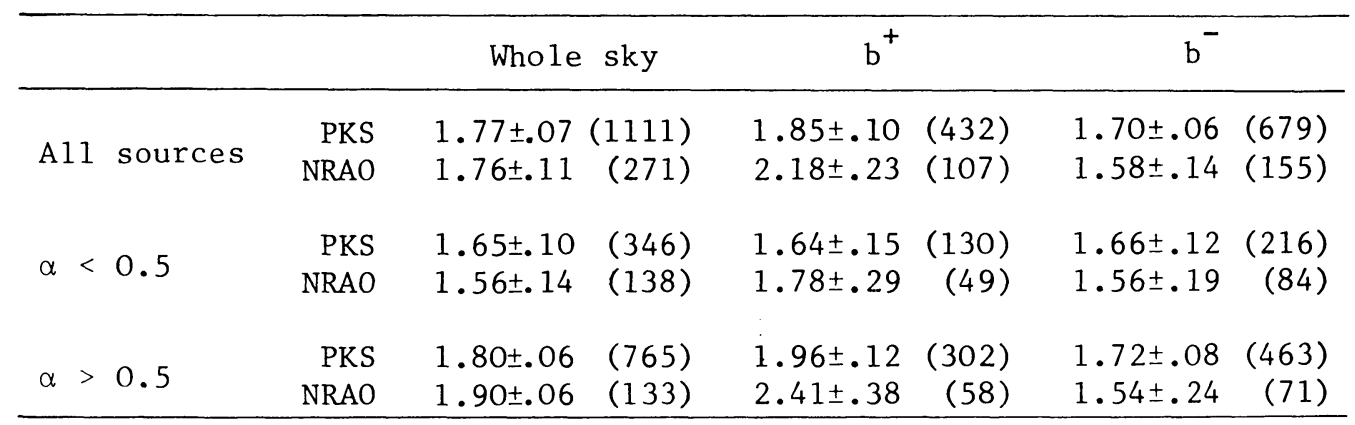

The effects found in the NRAO survey are still present in the $2700-\mathrm{MHz}$ data, but the weight of numbers has reduced the statistical significance to the level below which is is of interest. 


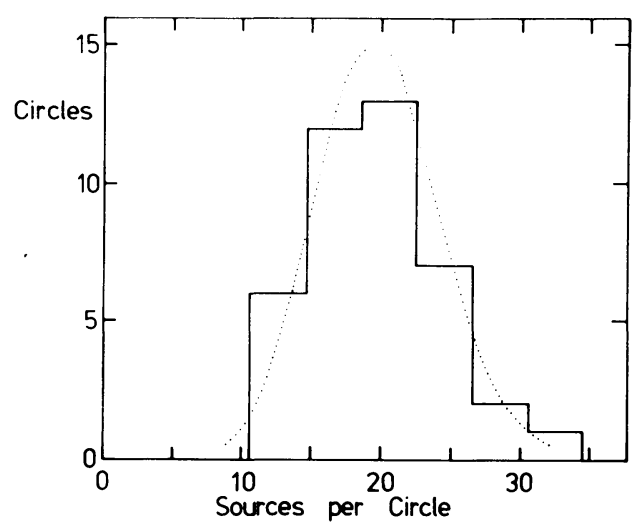

Fig. 7. Sources with $\mathrm{S}_{2700}>0.7 \mathrm{Jy}$ in 42 independent small circles $10^{8}$ in radius, $\sim 0.1 \mathrm{sr}$ in area.

Fig. 6 suggested anisotropies on the smaller scales of 0.1 to $1 \mathrm{sr}$. A simple binning analysis produced the result of Fig. 7; the numbers of sources in 42 independent small circles on the celestial sphere show no deviation from the Poissonian distribution expected for random distribution of the sources.

To examine clustering on all scales but the largest, the most powerful technique is that of power-spectrum analysis (PSA) as applied to the problem by Webster (1976). Several zones of the $2700 \mathrm{MHz}$ survey were subjected to this test (Webster, in press), and no deviation from isotropy is apparent. Since this analysis, more survey data have become available, completing to $\mathrm{S}_{2700}=0.35 \mathrm{Jy}$ a region bounded by R.A. $22^{\mathrm{h}}$ through to $05^{\mathrm{h}}$, dec. $+4^{\mathrm{O}}$ south to $-65^{\mathrm{O}}$. Such a "square" is highly suitable for PSA. The results (in Webster's notation) appear in Fig. 8,
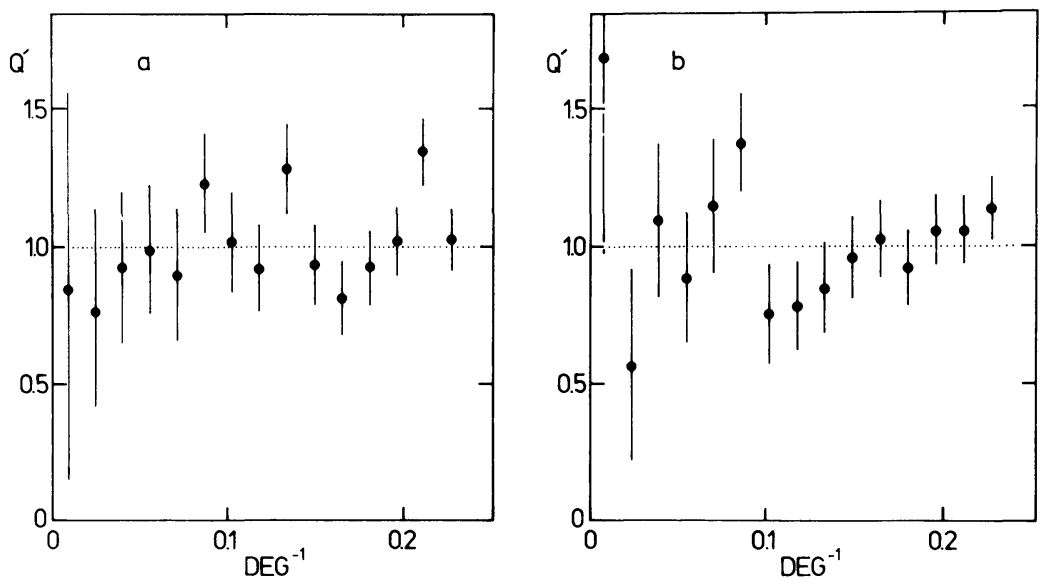

Fig. 8. PSA for R.A. $22^{\mathrm{h}}$ to $05^{\mathrm{h}}$, dec. $-65^{\mathrm{O}}$ to $+4^{\mathrm{O}}, \mathrm{S}_{2700}>0.35 \mathrm{Jy}$. (a) al1 836 sources, (b) the 225 flat-spectrum sources. 
and provide no indication that the sources are distributed in any way other than uniformly, randomly, and independently on the sky.

\section{CONCLUSIONS}

The absence of positive results in the clustering/anisotropy searches suggest (but do not prove) that the simplest hypothesis is correct: the sources are indeed randomly distributed on the sky, and the result of Fig. 6 and the differences between galactic hemispheres represent statistical mishaps. This is a working hypothesis in that it admits conventional analysis of the $270 \overline{\mathrm{MHz}} \mathrm{N}(\mathrm{S})$ relation. This relation is now defined down to $2 \times 10^{5} \mathrm{sr}^{-1}$, is continuous, and shows the same general features as low-frequency $N(S)$ relations. There is, however, the qualitative difference - the greatly extended region of turnover which reflects the increasing influence of flat-spectrum sources with increasing survey frequency, and implies that luminosity functions and/ or evolution for these sources must differ from those of the steepspectrum population.

I thank John Bolton, Ann Savage, Alan Wright and Jenny Trett for data in advance of publication, and Adrian Webster for help with the PSA.

\section{RE FERENCES}

Condon, J.J. and Jauncey, D.L.: 1974, Astron. J. 79, 437.

Fomalont, E.B., Bridle, A.H. and Davis, M.M.: 1974, Astron. Astrophys. $\underline{36}, 273$.

Jauncey, D.L.: 1975, Ann. Rev. Astron. Astrophys. 13, 23.

Pauliny-Toth, I.I.K. and Kellermann, K.I.: 1972, Astron. J. 77, 797. Shimmins, A.J., Bolton, J.G. and Wall, J.V.: 1968, Nature $21 \overline{7}, 818$. Wa11, J.V.: 1972, Australian J. Phys. Astrophys. Supp1. No. 24. Wa11, J.V.: 1975, Observatory 95, 196.

Wall, J.V. and Cooke, D.J.: 1975, Monthly Notices Roy. Astron. Soc. $171,9$.

Webster, A.S .: 1976, Monthly Notices Roy. Astron. Soc. 175, 61.

Yahil, A.: 1972, Astrophys. J. 178, 45.

\section{DISCUSSION}

van der Laan: Have you done any nearest neighbour analysis or triple and other compact group tests?

WaIZ: No, but the power spectrum analysis encompasses such tests and is considerably more powerful, as Webster has shown.

Kellermann: Is the galactic hemisphere difference less significant in the $2700 \mathrm{MHz}$ survey than in the $5000 \mathrm{MHz}$ survey?

Wall: Yes, and we have tried various flux density cut-off levels. 